\title{
Reliability and Validity of SymTrak, a Multi-Domain Tool for Monitoring Symptoms of Older Adults with Multiple Chronic Conditions
}

\author{
Patrick O. Monahan, $P h D^{1,2}$, Kurt Kroenke, $M D^{7,3,4}$, Christopher M. Callahan, MD ${ }^{1,4,5}$, \\ Tamilyn Bakas, $P h D^{6}$, Amanda Harrawood, $B S^{5}$, Phillip Lofton, $M P H^{5}$, Danielle Frye, $B S^{5}$, \\ Claire Draucker, $P h D^{7}$, Timothy Stump, $M S^{7}$, Debra Saliba, $M D^{8}$, James E. Galvin, $M D, M P H^{9}$, \\ Amanda Keegan ${ }^{5}$, Mary G. Austrom, PhD ${ }^{10}$, and Malaz Boustani, MD, MPH
}

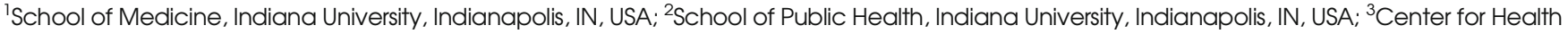
Information and Communication, VA HSR\&D, Washington, DC, USA; ${ }^{4}$, Regenstrief Institute, Inc., Indianapolis, IN, USA; ${ }^{5}$ Center for Aging Research, Indiana University, Indianapolis, IN, USA; ${ }^{6}$ College of Nursing, University of Cincinnati, Cincinnati, CA, USA; ${ }^{7}$ School of Nursing, Indiana University, Indianapolis, IN, USA; ${ }^{8}$ Borun Center and Veterans Administration Los Angeles, University of California, Los Angeles, CA, USA; ${ }^{9}$ Charles E. Schmidt College of Medicine, Florida Atlantic University, Boca Raton, FL, USA; ${ }^{10}$ Department of Psychiatry, Indiana University, Indianapolis, IN, USA.
\end{abstract}

BACKGROUND: A reliable and valid clinically practical multi-domain self-report and caregiver-report tool is needed for tracking actionable symptoms in primary care for elderly patients with multiple chronic conditions (MCCs). OBJECTIVE: Assess internal consistency reliability, testretest reliability, construct validity, and sensitivity to change for SymTrak.

DESIGN AND PARTICIPANTS: Among 600 (200 patientcaregiver dyads, 200 patients without an identified caregiver) participants, SymTrak was telephone intervieweradministered at baseline and 3-month follow-up, and at $24 \mathrm{~h}$ post-baseline for assessing test-retest reliability in a random subsample of 180 (60 dyads, 60 individual patients) participants.

MAIN MEASURES: Demographic questions, SymTrak, Health Utility Index Mark 3 (HUI3).

KEY RESULTS: Exploratory factor analysis indicated a single dominant dimension for SymTrak items for both patients and caregivers. Coefficient alpha and 24-h testretest reliability, respectively, were high for the 23-item SymTrak total score for both patient-reported $(0.85$; $0.87)$ and caregiver-reported $(0.86 ; 0.91)$ scores. Construct validity was supported by monotone decreasing relationships between the mean of SymTrak total scores across the poor-to-excellent categories of physical and emotional general health, and by high correlations with HUI3 overall utility score, even after adjusting for demographic covariates (standardized linear regression coefficient $=-0.84$ for patients; -0.70 for caregivers). Threemonth change in the SymTrak total score was sensitive to detecting criterion standard 3-month reliable change categories (Improved, Stable, Declined) in HUI3-based health-related quality of life, especially for caregiverreported scores.

Electronic supplementary material The online version of this article (https://doi.org/10.1007/s11606-018-4780-4) contains supplementary material, which is available to authorized users.

Received May 2, 2018

Revised September 18, 2018

Accepted November 27, 2018

Published online April 18, 2019
CONCLUSIONS: SymTrak demonstrates good internal consistency and test-retest reliability, construct validity, and sensitivity to change over a 3-month period, supporting its use for monitoring symptoms for older adults with MCCs.

KEY WORDS: primary care; psychometrics; chronic disease; aging; selfmanagement; symptoms.

J Gen Intern Med 34(6):908-14

DOI: $10.1007 / \mathrm{s} 11606-018-4780-4$

(c) Society of General Internal Medicine 2019

\section{INTRODUCTION}

SymTrak is a tool for monitoring clinically actionable symptoms that are relevant to multiple chronic conditions (MCCs). The development of SymTrak ${ }^{1}$ was important because elderly patients attending primary care clinics often have MCCs, ${ }^{2}$ concomitant complex health care needs, high health care costs, ${ }^{3}$ and various somatic, emotional, and cognitive symptoms. ${ }^{4,5}$ SymTrak is focused on symptoms that are not disease specific. Symptoms presented in primary care often do not yield a disease-based explanation, ${ }^{6}$ and yet these symptoms predict health care utilization and costs, quality of life, work disability, and mortality. ${ }^{4,5,7-11}$ For example, sleep disturbance, pain, anxiety, depression, and low energy/fatigue are prevalent, chronic, disabling, and under-treated symptoms that co-occur, ${ }^{12-14}$ interact synergistically, and adversely impact health and treatment response, which suggests clinicians should address multiple symptoms simultaneously. ${ }^{6}, 12,14,15$ A focus on clinically actionable symptoms is supported by the fact that symptoms account for over half of all US outpatient visits annually. ${ }^{10}$ Furthermore, some interventions are effective for more than one symptom category, ${ }^{6}$ making it wise to monitor the frequency or severity of symptoms with a tool that can improve diagnosis, ${ }^{16}$ guide treatment, and monitor treatment response. ${ }^{4}$ Developing a caregiver-report version was 
important because up to $57 \%$ of elderly patients are accompanied by an informal caregiver during physician visits. ${ }^{17}$

The development of SymTrak has been previously described. ${ }^{1}$ Online Appendix 1 shows the item-level similarities and differences in wording and formatting between SymTrak and legacy instruments. The present paper reports findings from a validation study in 600 participants. Internal consistency and test-retest reliability, construct validity, and sensitivity to change from baseline to 3 months were assessed.

\section{METHODS}

\section{Setting and Sample}

The 600 participants (200 patient-caregiver dyads and 200 non-dyadic patients without an identified caregiver) were recruited from an academic-affiliated primary care network of clinics. Patients with and without caregivers were equally sampled to investigate whether SymTrak was psychometrically sound in both groups. The study was approved by the institutional review board and all participants provided written informed consent. Patient inclusion criteria were the following: (1) age $\geq 65$ years, (2) $\geq$ one primary care visit in the past 12 months, (3) $\geq$ one chronic condition according to medical records, and (4) for those participants who had an identified informal caregiver, the caregiver had to be $\geq 21$ years of age and willing to participate in the study. Patient exclusion criteria were permanent residency at a long-term care facility or presence of a serious mental illness such as bipolar disorder or schizophrenia.

\section{Measures}

Participants $(N=600)$ completed a brief survey by interview consisting of demographics, SymTrak, and the Health Utility Index Mark 3 (HUI3), ${ }^{18}$ at baseline and 3 months post-baseline. A subsample of 60 patient-caregiver dyads and 60 nondyadic individual patients also were re-interviewed $24 \mathrm{~h}$ after baseline $(n=180)$. All interviews were telephoneadministered by research assistants.

The HUI3 is a general preference-based measure of health-related quality of life (HRQOL) for which the overall utility score of 0 represents death and 1 represents perfect health. A criterion standard for 3-month reliable HRQOL change groups (Decline, Stable, or Improve) was formulated based on \pm 1 standard error of measurement (SEM) in the HUI3 overall utility score. The HUI3 SEM was calculated as $1 \mathrm{SD}$ of the change in the HUI3 overall utility score from baseline to 3 months, multiplied by the square root of 1 minus the HUI3 24-h test-retest reliability coefficient. The value of 1 SEM equaled 0.092 for nondyadic patients, 0.90 for dyadic patients, and 0.085 for caregivers. A value of 0.089 was used for 1 SEM because it was between these values and it was the value for 1 SEM for all patients combined.

SymTrak consists of 23 items focused on clinically actionable symptoms measured on the following scale: $0=$ Never, $1=$ Sometimes, $2=$ Often, $3=$ Always. Two general health items (measured as Poor, Fair, Good, Very Good, Excellent) were assessed separately for physical and emotional health, and are included in the tool to provide researchers with validity correlations and to support clinical interpretations because nurses and physicians reported during focus groups in the SymTrak developmental study ${ }^{1}$ that they appreciated these general health ratings as additional information for gauging the patient's overall health.

\section{Analysis}

Exploratory factor analysis (EFA) was performed using the nonlinear ordered probit link for ordinal categorical items and WLSMV estimation in MPLUS software. The EFA was performed separately for patient- and caregiver-reported data, and results were explored using VARIMAX and PROMAX rotation. Cronbach's alpha was used to assess internal consistency reliability. The absolute-agreement version of the intra-class correlation coefficient (ICC) was used to assess test-retest reliability, with occasions specified as a random effect. For sensitivity to 3-month change, the mean change and withingroup standardized response mean (SRM) effect size (mean change divided by SD of change) were computed for SymTrak total scores, separately for each of three reliable HRQOL change groups (Decline, Stable, or Improve). A general linear model (GLM) was used to compare SymTrak change scores by testing the overall difference between all three HUI3 change groups using the omnibus $\mathrm{F}$ test, and then testing all pairwise differences (e.g., Decline vs Improve) using TukeyKramer post hoc tests with family-wise type I error controlled at 0.05 . Linear regression was used to test the concurrent predictive validity between baseline SymTrak scores and the dependent variable of baseline HUI3 overall utility scores (0100 metric) while adjusting for the patient's demographic characteristics. All tests were two-sided using 0.05 for alpha.

\section{RESULTS}

\section{Participant Characteristics}

Patients and caregivers were diverse with respect to race, education, income, and marital status (Table 1). Patients and caregivers from recruited dyads were allowed to remain in the study if their partner subsequently decided not to participate; thus, the number of caregivers $(n=203)$ is slightly greater than the number of dyadic patients $(n=200)$. The majority of caregivers were either a child $(43 \%)$ or a spouse/partner $(36 \%)$ of the dyadic patients (Table 1).

Compared to patients without an identified caregiver, patients with a recruited caregiver were significantly older by an 
Table 1 Participant Characteristics

\begin{tabular}{|c|c|c|c|}
\hline & $\begin{array}{l}\text { Patients } \\
\text { without } \\
\text { caregivers }\end{array}$ & $\begin{array}{l}\text { Patients } \\
\text { with } \\
\text { caregivers }\end{array}$ & Caregivers \\
\hline Characteristic & $(N=200)$ & $(N=200)$ & $(N=203)$ \\
\hline $\begin{array}{l}\text { Age, mean (SD); } \\
\text { median; range }\end{array}$ & $\begin{array}{l}73.5(6.2) \\
72 ; 65-95\end{array}$ & $\begin{array}{l}75.2(6.9) \\
74 ; 65-95\end{array}$ & $\begin{array}{l}59.3(12.8) \\
60 ; 22-86\end{array}$ \\
\hline \multicolumn{4}{|c|}{ Age categories, no. $(\%)$} \\
\hline$<50$ & $0(0.0)$ & $0(0.0)$ & $46(23.1)$ \\
\hline $50-64$ & $0(0.0)$ & $0(0.0)$ & $79(39.7)$ \\
\hline $65-74$ & $123(62.1)$ & $104(53.3)$ & $56(28.1)$ \\
\hline$>=75$ & $75(37.9)$ & $91(46.7)$ & $18(9.1)$ \\
\hline Female sex, no. $(\%)$ & $152(76.0)$ & $139(69.5)$ & $152(74.9)$ \\
\hline \multicolumn{4}{|l|}{ Race, no. (\%) } \\
\hline White & $75(37.5)$ & $97(48.5)$ & $99(48.8)$ \\
\hline Black & $119(59.5)$ & $97(48.5)$ & $100(49.3)$ \\
\hline Other & $6(3.0)$ & $6(3.0)$ & $4(1.9)$ \\
\hline Hispanic or Latino & $0(0.0)$ & $4(2.0)$ & $5(2.5)$ \\
\hline \multicolumn{4}{|c|}{ Ethnicity, no. (\%) } \\
\hline \multicolumn{4}{|c|}{ Highest level of education, no. (\%) } \\
\hline $\begin{array}{l}<\text { High school } \\
\text { graduate }\end{array}$ & $59(29.8)$ & $68(34.0)$ & $33(16.3)$ \\
\hline $\begin{array}{l}\text { High school } \\
\text { graduate }\end{array}$ & $65(32.8)$ & $51(25.5)$ & $67(33.0)$ \\
\hline $\begin{array}{l}\text { Some college or } \\
\text { higher }\end{array}$ & $74(37.4)$ & $81(40.5)$ & $103(50.7)$ \\
\hline \multicolumn{4}{|c|}{ Total household income, past year, no. (\%) } \\
\hline$<\$ 15,000$ & $128(64.0)$ & $79(39.5)$ & $61(30.0)$ \\
\hline$\$ 15,000-\$ 30,000$ & $52(26.0)$ & $54(27.0)$ & $54(26.6)$ \\
\hline$>\$ 30,000$ & $14(7.0)$ & $53(26.5)$ & $73(36.0)$ \\
\hline Unknown & $6(3.0)$ & $14(7.0)$ & $15(7.4)$ \\
\hline \multicolumn{4}{|c|}{ Marital status, no. (\%) } \\
\hline $\begin{array}{l}\text { Married or living } \\
\text { together }\end{array}$ & $39(19.5)$ & $77(38.5)$ & $108(53.2)$ \\
\hline Widowed & $59(29.5)$ & $71(35.5)$ & $13(6.4)$ \\
\hline $\begin{array}{l}\text { Divorced or } \\
\text { separated }\end{array}$ & $80(40.0)$ & $38(19.0)$ & $41(20.2)$ \\
\hline Never married & $22(11.0)$ & $13(6.5)$ & $41(20.2)$ \\
\hline Unknown & $0(0.0)$ & $1(0.5)$ & $0(0.0)$ \\
\hline \multicolumn{4}{|c|}{ Relationship to patient, no. (\%) } \\
\hline Spouse or partner & $\mathrm{N} / \mathrm{A}$ & $\mathrm{N} / \mathrm{A}$ & $73(36.0)$ \\
\hline Child & & & $88(43.4)$ \\
\hline Sibling & & & $16(7.9)$ \\
\hline Grandchild & & & $5(2.5)$ \\
\hline Parent & & & $3(1.5)$ \\
\hline Other & & & $18(8.9)$ \\
\hline \multicolumn{4}{|c|}{ Patient's general physical health* } \\
\hline Poor & $20(10.0)$ & $23(11.5)$ & $28(13.9)$ \\
\hline Fair & $81(40.5)$ & $89(44.5)$ & $85(42.1)$ \\
\hline Good & $71(35.5)$ & $56(28.0)$ & $67(33.2)$ \\
\hline Very Good & $26(13.0)$ & $23(11.5)$ & $21(10.4)$ \\
\hline Excellent & $2(1.0)$ & $9(4.5)$ & $1(0.5)$ \\
\hline \multicolumn{4}{|c|}{ Patient's general emotional health* } \\
\hline Poor & $9(4.5)$ & $7(3.6)$ & $8(4.0)$ \\
\hline Fair & $61(30.5)$ & $48(24.5)$ & $65(32.5)$ \\
\hline Good & $78(39.0)$ & 93 (47.4) & $84(42.0)$ \\
\hline Very Good & $45(22.5)$ & $31(15.8)$ & $31(15.5)$ \\
\hline Excellent & $7(3.5)$ & $17(8.7)$ & $12(6.0)$ \\
\hline
\end{tabular}

*The stem for the single-item general health ratings was "In general, how would you describe your ['your loved ones' for caregiver-report] physical health [and emotional health]." N/A = not applicable. The two patient groups were compared on continuous age, unordered categorical variables, and ordinal categorical variables using the two-sided $t$ test, Pearson chi-square test, and Mantel $1 \mathrm{df}$ test of trend, respectively

average of 2 years $(p=0.01)$ but also more likely to be married or living together $(p<0.0001)$ and with higher household income $(p<0.0001)$, although income was low for all groups (Table 1). Given these demographic characteristics, it is not surprising that the two patient groups did not differ significantly on HUI3 functional status, SymTrak total symptom scores, or poor-to-excellent general health ratings. African Americans were well represented in this sample, comprising $49 \%$ of dyadic patients and caregivers, and $60 \%$ of patients without caregivers. In all three samples, the majority of participants reported the patient's health to be in either fair or good health on both emotional and physical health (Table 1).

\section{Data Distribution}

All 23 items on SymTrak received endorsement for all 4 response options by the patients (Table 2). The response option distribution was similar for patients with and without an identified caregiver; therefore, all patients were combined for Table 2. A majority of patients endorsed the Never or Sometimes options for most items; however, for nearly half of the tool (10 items), a meaningful percentage (17 to 52\%) of patients endorsed either Often or Always (Table 2). Thus, SymTrak exhibited reasonable spread in its item response scores. Patients reported a relatively higher level of endorsement (indicated by item means of 1.0 or greater) for items assessing fatigue (no. 1), sleep disturbance (no. 2), pain (no. 3-no. 5), and mobility (no. 12). These item statistics were similar when the symptoms of patients were reported by their caregivers (Online Appendix 2). The SymTrak total score has a possible range from 0 to 69 and was approximately normally distributed in all three participant groups (Online Appendix 3). Concordance between dyadic patients and their caregivers was reasonably close for most items, with a similar mean item score and a kappa coefficient representing moderate agreement (Online Appendix 4).

\section{Factor Analysis}

The EFA scree plot revealed a single dominant dimension for patients and caregivers (Fig. 1). Loadings from the one-factor solution were good (i.e., > 0.40) for 20 items, ranging from 0.43 to 0.70 ; for three items (no. 7, no. 11, and no. 20), loadings were lower but adequate (between 0.30 and 0.40 ; Table 2). Thus, factor analysis supported the validity of using the total score as an overall summary of symptoms. The validity of additional subscale scoring was investigated by exploring rotated factor loadings for the 2-, 3-, 4-, and 5factor EFA solutions; however, none of the factor models, regardless of rotation method, offered a meaningfully clearer factor structure (i.e., higher loadings without cross-loadings on other domains) compared to the one-factor model. For example, although cognitive items often clustered together, and emotional items usually clustered together, several physical items demonstrated cross-loadings with the cognitive or emotional domains in all rotated factor solutions.

\section{Reliability}

Coefficient alpha for the SymTrak total score was high for non-dyadic patients $(0.85)$, dyadic patients $(0.86)$, and caregivers (0.86). Test-retest reliability also revealed high 
Table 2 Patient-Reported SymTrak Item Distributions and Loadings $(N=400)$

\begin{tabular}{|c|c|c|c|c|c|c|c|c|c|}
\hline \multirow[t]{2}{*}{ SymTrak Items } & \multirow{2}{*}{$\begin{array}{l}\text { Item } \\
N\end{array}$} & \multirow{2}{*}{$\begin{array}{l}\text { Item } \\
\text { mean }\end{array}$} & \multirow{2}{*}{$\begin{array}{l}\text { Item } \\
\text { SD }\end{array}$} & \multicolumn{4}{|c|}{ Item Response \% } & \multirow{2}{*}{$\begin{array}{l}\text { 1-Factor } \\
\text { loadings }\end{array}$} & \multirow[t]{2}{*}{$r$} \\
\hline & & & & Never & Sometimes & Often & Always & & \\
\hline 1. Feeling tired or having low energy & 400 & 1.3 & 0.8 & 14 & 53 & 23 & 10 & 0.62 & 0.52 \\
\hline $\begin{array}{l}\text { 2. Trouble falling asleep or trouble staying } \\
\text { asleep }\end{array}$ & 400 & 1.0 & 1.0 & 35 & 42 & 13 & 10 & 0.43 & 0.37 \\
\hline 3. Pain interfering with daily activities & 400 & 1.3 & 1.1 & 29 & 35 & 19 & 17 & 0.69 & 0.53 \\
\hline 4. Pain in the back, arms, legs, or joints & 400 & 1.6 & 1.0 & 14 & 34 & 25 & 27 & 0.70 & 0.55 \\
\hline 5. Foot pain or foot numbness & 400 & 1.1 & 1.0 & 35 & 36 & 15 & 14 & 0.43 & 0.36 \\
\hline 6. Constipation or stomach problems & 400 & 0.8 & 0.8 & 42 & 41 & 12 & 5 & 0.51 & 0.42 \\
\hline 7. Trouble with urination & 400 & 0.4 & 0.8 & 71 & 20 & 5 & 4 & 0.31 & 0.24 \\
\hline 8. Shortness of breath & 400 & 0.9 & 0.9 & 40 & 43 & 9 & 8 & 0.61 & 0.53 \\
\hline 9. Chest pain & 400 & 0.3 & 0.6 & 73 & 24 & 2 & 1 & 0.49 & 0.34 \\
\hline 10. Trouble with vision & 398 & 0.9 & 1.0 & 39 & 42 & 7 & 12 & 0.44 & 0.37 \\
\hline 11. Trouble with hearing & 400 & 0.8 & 1.0 & 49 & 33 & 8 & 10 & 0.35 & 0.30 \\
\hline $\begin{array}{l}\text { 12. Trouble walking or trouble moving } \\
\text { around }\end{array}$ & 400 & 1.1 & 1.0 & 31 & 42 & 15 & 12 & 0.60 & 0.51 \\
\hline 13. Falling or tripping & 400 & 0.3 & 0.5 & 74 & 23 & 2 & 1 & 0.47 & 0.33 \\
\hline $\begin{array}{l}\text { 14. Less interest or less pleasure in doing } \\
\text { things }\end{array}$ & 399 & 0.7 & 0.8 & 43 & 47 & 6 & 4 & 0.70 & 0.54 \\
\hline 15. Feeling sad, down, or depressed & 400 & 0.6 & 0.7 & 55 & 38 & 5 & 2 & 0.68 & 0.46 \\
\hline 16. Poor appetite or overeating & 400 & 0.7 & 0.8 & 45 & 43 & 7 & 5 & 0.48 & 0.35 \\
\hline 17. Feeling nervous or anxious & 400 & 0.6 & 0.7 & 49 & 43 & 5 & 3 & 0.62 & 0.45 \\
\hline $\begin{array}{l}\text { 18. Worrying too much about different } \\
\text { things }\end{array}$ & 400 & 0.9 & 0.9 & 39 & 44 & 10 & 7 & 0.64 & 0.48 \\
\hline 19. Becoming easily annoyed or irritable & 399 & 0.6 & 0.7 & 48 & 42 & 7 & 3 & 0.56 & 0.41 \\
\hline $\begin{array}{l}\text { 20. Trouble taking medications in the right } \\
\text { dose at the right time }\end{array}$ & 400 & 0.3 & 0.6 & 76 & 20 & 1 & 3 & 0.32 & 0.20 \\
\hline 21. Trouble remembering appointments & 400 & 0.4 & 0.6 & 69 & 27 & 2 & 2 & 0.58 & 0.40 \\
\hline 22. Trouble concentrating on things & 400 & 0.7 & 0.7 & 45 & 48 & 5 & 2 & 0.66 & 0.50 \\
\hline 23. Memory Loss & 400 & 0.7 & 0.7 & 39 & 52 & 7 & 2 & 0.56 & 0.43 \\
\hline
\end{tabular}

Item stem: "Over the past two weeks, how often have you had problems with:". Item response options: $0=$ Never, $1=$ Sometimes, $2=$ Often, $3=$ Always. $r=$ corrected item-total Pearson correlation

agreement between baseline and 24-h retest values for nondyadic patients (0.87), dyadic patients (0.88), and caregivers (0.91). For readers interested in the reliability over all patients combined, internal consistency and test-retest reliability were 0.85 and 0.87 , respectively.

\section{Construct Validity}

SymTrak demonstrated a dose-response relationship with the single-item general health ratings (Online Appendix 5). Specifically, the mean SymTrak total score generally decreased as patient health rating categories increased from Poor to Excellent (the two exceptions occurred in rating categories with less than 10 participants). This dose-response relationship was evident for both physical and emotional general health ratings, and for both patient-reported and caregiver-reported data.

Spearman correlations between the SymTrak total score and the HUI3 overall utility score were strong: -0.83 for nondyadic patients, -0.84 for dyadic patients, $(-0.84$ when disseminating for all patients combined), and -0.69 for caregivers. The scatterplot between SymTrak total score and HUI3 overall utility score revealed a reasonably linear relationship; as symptom burden decreased, preference-based HRQOL increased (Online Appendix 6). Baseline SymTrak total score was strongly associated with baseline HUI3 HRQOL after adjusting for demographic covariates (standardized linear regression beta coefficient $[\mathrm{STB}]=-0.84$ for each of the patient groups and -0.70 for caregivers; Table 3 ). The following is an example of interpreting the models in Table 3. For every $1 \mathrm{SD}$ increase in caregiver-reported SymTrak total score, the patient's caregiver-reported HUI3 utility score

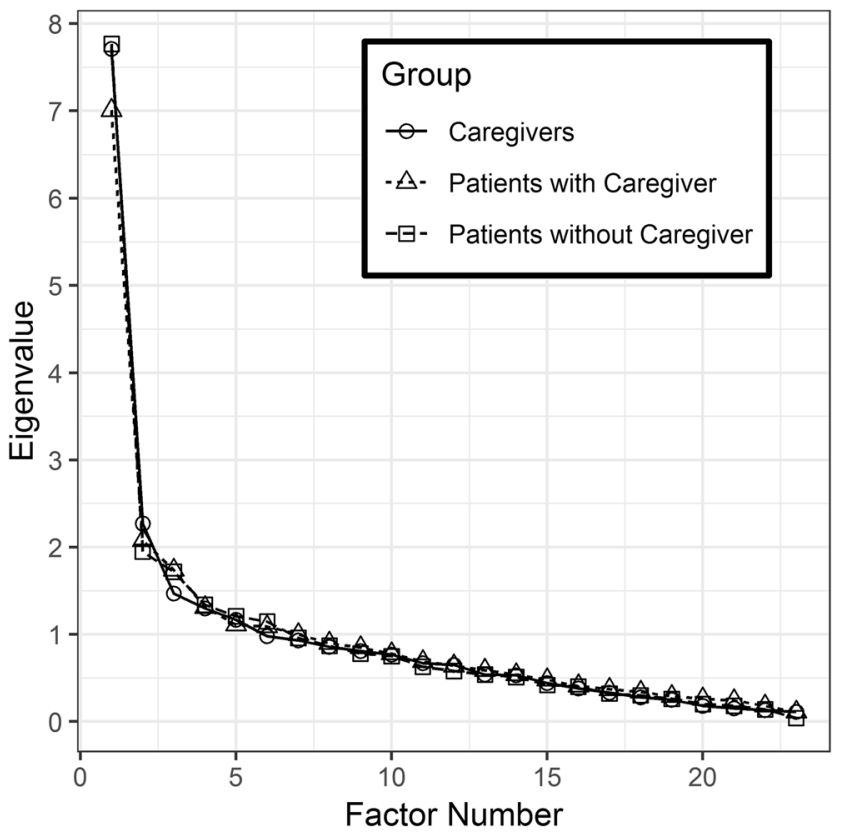

Fig. 1 Factor analysis scree plot. 
Table 3 SymTrak as a Predictor of Health Utility Index Mark 3 (HUI3)

\begin{tabular}{|c|c|c|c|c|c|c|}
\hline \multirow[b]{3}{*}{ Patient's baseline characteristics } & \multicolumn{6}{|c|}{ SymTrak symptoms and HUI3 HRQOL were assessed about the patients and reported by: } \\
\hline & \multicolumn{2}{|c|}{$\begin{array}{l}\text { Model } 1 \\
\text { Patients without } \\
\text { caregivers } \\
(N=195)\end{array}$} & \multicolumn{2}{|c|}{$\begin{array}{l}\text { Model } 2 \\
\text { Patients with caregivers } \\
(N=195)\end{array}$} & \multicolumn{2}{|c|}{$\begin{array}{l}\text { Model } 3 \\
\text { Caregivers } \\
(N=177)\end{array}$} \\
\hline & STB & $p$ value & STB & $p$ value & STB & $p$ value \\
\hline Age & -0.09 & 0.04 & -0.06 & 0.16 & -0.09 & 0.09 \\
\hline Sex, female vs male & 0.01 & 0.85 & -0.06 & 0.23 & -0.03 & 0.66 \\
\hline \multicolumn{7}{|l|}{ Race } \\
\hline Black vs White & 0.07 & 0.11 & -0.02 & 0.58 & -0.02 & 0.76 \\
\hline Other vs White & 0.07 & 0.09 & 0.01 & 0.73 & -0.04 & 0.49 \\
\hline \multicolumn{7}{|l|}{ Highest level of education } \\
\hline High school (HS) graduate vs $<$ HS & 0.06 & 0.18 & 0.03 & 0.46 & -0.05 & 0.40 \\
\hline Some college or higher vs $<$ HS & -0.04 & 0.37 & -0.02 & 0.71 & 0.08 & 0.23 \\
\hline \multicolumn{7}{|l|}{ Total household income } \\
\hline$\$ 15,000-\$ 30,000$ vs $<\$ 15,000$ & 0.02 & 0.61 & 0.04 & 0.37 & 0.08 & 0.21 \\
\hline$>\$ 30,000$ vs $<\$ 15,000$ & 0.05 & 0.25 & 0.08 & 0.16 & 0.16 & 0.04 \\
\hline Unknown vs $<\$ 15,000$ & 0.02 & 0.70 & 0.01 & 0.84 & 0.03 & 0.63 \\
\hline \multicolumn{7}{|l|}{ Marital status } \\
\hline Married/living together vs not & -0.01 & 0.83 & -0.07 & 0.17 & -0.07 & 0.29 \\
\hline SymTrak total score & -0.84 & $<0.0001$ & -0.84 & $<0.0001$ & -0.70 & $<0.0001$ \\
\hline
\end{tabular}

Each column of data (standardized regression coefficient [STB], $p$ value) represents a different multivariable linear regression model with HUI3 overall utility score as the dependent variable. The SymTrak and HUI3 instruments were assessed in this study about the patient's symptoms and the patient's preference-based health-related quality of life (HRQOL), respectively, and were reported by the patients in models 1 and 2 and reported by the caregivers in model 3. However, for this table, all demographics are about the patient and were reported by the patient, even for model 3. This was because the purpose of these models was to determine whether SymTrak-measured patient symptoms predict HUI3-measured patient HRQOL, for selfreported and caregiver-reported SymTrak and HUI3 scores, while holding constant the same demographics in all three models and adjusting for demographics that have the greatest theoretical impact on patient HRQOL, which are the patient's demographics even though the caregiver's demographics may contribute to a lesser degree to the patient's HRQOL. Therefore, model 3 uses a smaller sample size because it required patientreported demographics and caregiver-reported SymTrak and HUI3 data from intact dyads. Similarly, the SymTrak concordance results in Online Appendix 4 also required data from intact dyads. In contrast, data from patients and their caregivers who were no longer part of an intact dyad (i.e., because they were allowed to remain in the study even if their partner dropped out after dyadic recruitment and informed consent) were allowed to contribute to results in other tables and other Online Appendices in this article

decreased on average by $0.70 \mathrm{SD}$ after adjusting for the patient's age, sex, race, education, household income, and marital status (model 3). Older age and very low income significantly predicted poorer HRQOL but their impact on HRQOL was relatively small compared to the impact of SymTrak-measured symptoms (model $1, \mathrm{STB}_{\mathrm{age}}=-0.09$, $\mathrm{STB}_{\text {SymTrak }}=-0.84 ;$ model $3, \mathrm{STB}>\$ 30,000$ vs $<\$ 15,000=$ 0.16, STB $_{\text {SymTrak }}=-0.70$ ).

\section{Sensitivity to Change}

Validity was supported by the sensitivity-to-change analysis (Table 4). The SRM represents the magnitude of the change in the SymTrak total score over 3 months. For all three participant samples, the SRM was reasonably centered near 0 , as expected, for patients in the HUI3 Stable group (Table 4).

In addition, for patient-reported and caregiver-reported scores, the SRM for SymTrak was in the anticipated negative direction for the group of patients that declined in HRQOL and in the positive direction for the group of patients that improved in HRQOL. For all three samples, the omnibus $p$ value for comparing SymTrak change scores between the three HRQOL change groups was significant, and the pairwise difference between HRQOL Decline and Improve groups (D vs I) was significant (Table 4). Pairwise comparisons of the Decline and
Improve groups with the reference (Stable) group were not significant, except for the caregiver-reported SymTrak total score which significantly differentiated between Decline and Stable HRQOL groups due to caregivers reporting a larger SymTrak SRM for the Decline group.

\section{DISCUSSION}

The SymTrak tool demonstrated an adequate spread of item response scores across its 23 symptom-focused items. The EFA revealed one dominant dimension for explaining the correlation among the SymTrak item scores, with adequately strong factor loadings for the one-factor solution, for both patient-reported and caregiver-reported data. The internal consistency reliability and test-retest reliability coefficients were very high for the SymTrak total score. Perhaps future factor analyses in other samples will reveal valid subscale scoring options despite the fact that EFA in this data, using state-ofthe-art nonlinear factor analysis, did not reveal clean subscales. Physical symptoms (e.g., sleep disturbance, fatigue, pain) often cluster with anxiety, depression, and cognitive symptoms in primary care populations. It is possible that these symptoms correlate across domains even more highly in populations with MCCs. The baseline cross-sectional analyses 
Table 4 Sensitivity-to-Change Validity of SymTrak

Criterion Standard HRQOL 3-Month Reliable Change Groups

\begin{tabular}{|c|c|c|c|c|c|c|c|c|c|c|c|}
\hline \multicolumn{4}{|c|}{ HUI3 Decline (D) } & \multicolumn{4}{|c|}{ HUI3 Stable (S) } & \multicolumn{4}{|c|}{ HUI3 Improve (I) } \\
\hline & Panel & $\mathrm{CumT}_{\mathrm{S}}$ & Chor & & Nepuit & D he & tionts & O & givers ( & $=17$ & \\
\hline $\mathrm{N}$ & Mean & SD & SRM & $\mathrm{N}$ & Mean & SD & SRM & $\mathrm{N}$ & Mean & SD & SRM \\
\hline 52 & -0.77 & 5.20 & -0.15 & 72 & 0.82 & 5.28 & 0.16 & 46 & 1.93 & 5.59 & 0.35 \\
\hline
\end{tabular}

GLM: Omnibus, $p=0.04$; D vs. $S, p=0.23$; D vs. $I, p=0.04 ; S$ vs $I, p=0.50$

Panel B. SymTrak Change Scores Reported by Patients with Caregivers $(N=170)$

\begin{tabular}{|c|c|c|c|c|c|c|c|c|c|c|c|}
\hline $\mathrm{N}$ & Mean & SD & SRM & $\mathrm{N}$ & Mean & SD & SRM & $\mathrm{N}$ & Mean & SD & SRM \\
\hline 61 & -1.33 & 5.42 & -0.25 & 56 & 0.23 & 5.06 & 0.05 & 53 & 2.11 & 6.41 & 0.33 \\
\hline
\end{tabular}

GLM: Omnibus, $p=0.01 ; D$ vs. $S, p=0.29 ; D$ vs. I, $p=0.01 ; S$ vs I, $p=0.19$

Panel C. SymTrak Change Scores Reported by Caregivers $(\mathrm{N}=164)$

\begin{tabular}{|c|c|c|c|c|c|c|c|c|c|c|c|}
\hline $\mathrm{N}$ & Mean & SD & SRM & $\mathrm{N}$ & Mean & SD & SRM & $\mathrm{N}$ & Mean & SD & SRM \\
\hline 45 & -4.33 & 7.33 & -0.59 & 66 & -0.53 & 6.17 & 0.09 & 53 & 2.25 & 6.36 & 0.35 \\
\hline
\end{tabular}

GLM: Omnibus, $p<0.001$; D vs. $S, p=0.01$; D vs. I, $p<0.001 ; S$ vs I, $p=0.06$

The standardized response mean (SRM) effect size equals the mean change for SymTrak total score (baseline- 3 months) divided by the SD of change scores. A positive effect size indicates a decrease in the SymTrak symptom total score which represents improvement in symptoms of patients. A negative effect size represents a worsening in symptoms. The HUI3 overall utility score was used to measure preference-based health-related quality of life (HRQOL), for which a higher score is better. The HUI3 Decline, Stable, and Improve groups were defined using a threshold of \pm 1 standard error of measurement (SEM) of change on HUI3 overall utility score (i.e., a change of 0.089). Each panel (A, B, C) of values is from a separate general linear model (GLM) where, for the purpose of this analysis, HUI3 reliable change groups were the independent variables and the change score of the SymTrak total score was the dependent variable

supported the construct validity of the SymTrak total score including dose-response relationships across poor-toexcellent categories of physical and emotional general health ratings. Also shown were high correlations with the HUI3 overall utility score, regardless of whether the patients' symptoms and HRQOL were reported by the patients themselves or by their informal caregivers. When demographics were included in a linear regression model for predicting the HUI3 overall utility score, the SymTrak total score was much more strongly associated with preference-based HRQOL than were the patient's demographic variables including age. Importantly, this finding occurred in elderly patients with a wide age range (from 65 to 95 ) in which $41 \%$ were 75 or older.

An important aspect of validity, especially for a tool developed primarily for monitoring treatment response, is sensitivity to change. The SymTrak total score demonstrated good sensitivity to change, especially considering the short 3-month interval. For both patient-reported and caregiver-reported data, the SymTrak total score was able to distinguish between the three HUI3-defined groups of reliable change in HRQOL, most notably between declining and improving HUI3-based HRQOL. However, only caregiver-reported SymTrak scores were sensitive enough to correlate with declining HRQOL and thereby detect the difference between declining and stable HRQOL over a brief 3-month period. This suggests that caregiver-reported SymTrak data may be particularly useful for monitoring patients' symptoms during times of suspected declines. This should be further investigated in future longitudinal studies in which caregiver- and patient-reported SymTrak scores are compared to each other regarding their sensitivity for earlier detection of clinically verified declines in HRQOL and other measures of functioning.

\section{Limitations}

Because the sample only included adults 65 and older, generalizability to individuals less than 65 needs to be investigated in future studies. Second, sensitivity to change of SymTrak should be studied over a longer interval than 3 months and with additional validators besides the HUI3. Also, 
responsiveness to treatment should be tested in clinical trials and other prospective studies aimed at reducing symptom burden for patients with MCCs. Third, our sample of older adults attending primary care with chronic conditions was generally cognitively intact; therefore, the performance of SymTrak in patients with mild to moderate cognitive impairment should be evaluated. Fourth, all interviews were administered by telephone; therefore, we could not compare with other modes of administration. However, a previous study proved that self-administration of SymTrak in clinics, for patients aged 65 and older and their informal caregivers, is psychometrically sound and efficient for both patient-reported and caregiver-reported scores. ${ }^{1}$ The use of SymTrak scores (both baseline and change over time) to predict future health care utilization would further inform predictive validity.

\section{Research and Clinical Implications}

The SymTrak total score was approximately normally distributed. This is a convenient advantage in parametric statistical models especially when the score is used as a dependent variable. For example, SymTrak may be useful for assessing response to treatment in randomized controlled trials or in observational correlational studies. In populations with MCCs, and where treatment of one symptom synergistically affects response on other symptoms, ${ }^{6,12,14,15}$ the SymTrak total score may serve as a useful overall measure of treatment outcome in research and clinical practice, especially given its promising sensitivity to detect change in HRQOL over a brief 3 -month period. SymTrak performed psychometrically well in all three samples (i.e., both patient groups and caregivers), which represents a strength of the scale for research and clinical practice.

Corresponding Author: Patrick O. Monahan, $\mathrm{PhD}$; School of Medicine, Indiana University, Indianapolis, IN, USA (e-mail: pmonahan@iu.edu).

Funding This work was supported by a National Institute on Aging RO1 award to P.O.M. (RO1 AG043465).

\section{Compliance with Ethical Standards:}

Conflict of Interest: Patrick O. Monahan is Chief Technology Officer and has $2.5 \%$ equity ownership (valued at \$2500) in a for-profit company called RestUp. Malaz Boustani is a deferred-paid advisor with 5\% equity (valued at \$5000) in the same company, RestUp. The purpose of RestUp is to use the Internet and mobile technology to connect caregivers and care seekers. The RestUp caregivers are paid hourly, as 1099 contractors, by care seekers, and RestUp earns its income by receiving a percentage of each hour worked. The present paper has no overlap with the RestUp company; the SymTrak tool developed in the paper is not used in the RestUp company; and none of the activities of the RestUp company are involved in any way with this paper or the SymTrak tool. All other authors have no financial or non-financial interests.
Disclaimer: The sponsor had no role in study design; in the collection, analysis, and interpretation of data; in the writing of the report; or in the decision to submit the article for publication. The views expressed in this article are those of the authors and do not necessarily represent the views of the National Institute on Aging.

\section{REFERENCES}

1. Monahan PO, Kroenke $\mathbf{K}$, Callahan CM, et al. Development and feasibility of SymTrak, a multi-domain tool for monitoring symptoms of older adults in primary care. J Gen Intern Med. 2019. https://doi.org/ $10.1007 / \mathrm{s} 11606-018-4772-4$

2. Schneider KM, O'Donnell BE, Dean D. Prevalence of multiple chronic conditions in the United States' Medicare population. Health Qual Life Outcomes. 2009;7:82.

3. Leroy L, Bayliss E, Domino M, et al. The Agency for Healthcare Research and Quality Multiple Chronic Conditions Research Network: overview of research contributions and future priorities. Med Care. 2014;52 Suppl 3:S15-22.

4. Sha MC, Callahan CM, Counsell SR, Westmoreland GR, Stump TE, Kroenke $\mathbf{K}$. Physical symptoms as a predictor of health care use and mortality among older adults. Am J Med. 2005;118:301-6.

5. Callahan CM, Weiner M, Counsell SR. Defining the domain of geriatric medicine in an urban public health system affiliated with an academic medical center. J Am Geriatr Soc. 2008;56:1802-6.

6. Kroenke K. A practical and evidence-based approach to common symptoms: a narrative review. Ann Intern Med. 2014;16:579-86.

7. Kessler RC, Zhao S, Katz SJ, et al. Past-year use of outpatient services for psychiatric problems in the National Comorbidity Survey. Am J Psychiatry. 1999;156:115-23.

8. Korten AE, Jorm AF, Jiao Z, et al. Health, cognitive, and psychosocial factors as predictors of mortality in an elderly community sample. J Epidemiol Community Health. 1999;53:83-8.

9. Kroenke $\mathbf{K}$, Spitzer $\mathbf{R}$, Williams JB, et al. Physical symptoms in primary care: predictors of psychiatric disorders and functional impairment. Arch Fam Med. 1994;3:774-9.

10. Kroenke K. Studying symptoms: sampling and measurement issues. Ann Intern Med. 2001;134:844-53.

11. Kroenke K. Patients presenting with somatic complaints: epidemiology, psychiatric comorbidity and management. Int J Methods Psychiatr Res. 2003; 12:34-43.

12. Davis LL, Kroenke $\mathbf{K}$, Monahan $\mathbf{P}$, Kean J, Stump TE. The SPADE symptom cluster in primary care patients with chronic pain. Clin $\mathrm{J}$ Pain. 2016;32:388-93.

13. Kroenke K, Bair MJ, Damush TM, et al. Optimized antidepressant therapy and pain self-management in primary care patients with depression and musculoskeletal pain: a randomized controlled trial. JAMA. 2009;301:2099-110.

14. Kroenke K, Wu J, Bair MJ, Krebs EE, Damush TM, Tu W. Reciprocal relationship between pain and depression: a 12-month longitudinal analysis in primary care. J Pain. 2011;12:964-73.

15. Aktas A, Walsh D, Rybicki L. Symptom clusters: myth or reality? Palliat Med. 2010;24:373-85.

16. Komaroff AL. 'Minor' illness symptoms: the magnitude of their burden and of our ignorance. Arch Intern Med. 1990;150:1586-7.

17. Adelman RD, Greene MG, Ory MG. Communication between older patients and their physicians. Clin Geriatr Med. 2000;16:1-24, vii.

18. Horsman J, Furlong W, Feeny D, Torrance G. The Health Utilities Index (HUI): concepts, measurement properties and applications. Health Qual Life Outcomes. 2003;1:54.

Publisher's Note: Springer Nature remains neutral with regard to jurisdictional claims in published maps and institutional affiliations. 